\title{
Nível de Atividade Física como Estimador da Aptidão Física de Estudantes Universitários: Explorando a Adoção de Questionário Através de Modelagem Linear
}

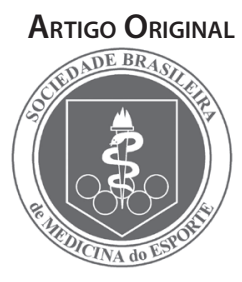

\author{
Physical Activity and Fitness in College Studients: Exploring Adoption \\ of a Questionnaire by Linear Modeling
}

Marcelo Conte

Aguinaldo Gonçalves ${ }^{2}$

Liciana Vaz de Arruda Silveira

Chalita $^{3}$

Luiz Carlos de Barros Ramalho²

1. Escola Superior de Educação Física de Jundiaí.

2. Universidade Estadual de

Campinas.

3. Universidade Estadual de São Paulo.

Endereço para correspondência: Marcelo Conte

Rua Laura Maiello Kook, 601 - apto 31 - Bloco 4 - Ipanema das Pedras CEP: $18052-580$ - Sorocaba/SP E-mail: conte@uirapuru.edu.br

Submetido em 01/08/2006 Versão final recebida em 08/01/2006 Aceito em 25/01/2008

\begin{abstract}
RESUMO
A classificação do nível de atividade física (NAF) tem-se revelado aspecto controvertido em Ciência do Esporte. Nesta perspectiva, o objetivo da presente investigação foi verificar a utilização de instrumento adaptado para classificação do NAF. Para tanto, foi desenvolvido estudo transversal seriado, considerando NAF como variável independente e a aptidão física como dependente. Identificaram-se como população de estudo calouros do curso de Medicina, em total de 290 pessoas. Foram coletados durante três anos subseqüentes, através de anamnese dirigida, informações a respeito do NAF e testes de capacidade aeróbia e muscular, para conhecer as variáveis de aptidão física (AF). A análise estatística foi realizada através do modelo Linear, sendo aplicado o teste $\mathrm{F}$ para avaliar o efeito das variáveis independentes, bem como a prova de Tukey para comparar as respectivas médias e o modelo de Poisson para verificar o efeito das variáveis dependentes, segundo nível de atividade física e sexo. Como principal resultado, destaca-se o fato de as pessoas que referiram maior NAF também apresentaram os melhores escores de AF indicando que a utilização do instrumento revelou-se coerente e compatível.
\end{abstract}

Palavras-chave: nível de atividade física, aptidão física, atividade física, questionário.

\section{ABSTRACT}

Classification of Physical Activity Level (PAL) is currently considered as a controversial issue inside Sport Science. The aim of study was verify the uses of an adapted questionnaire to classify PAL. The cross-serial essay was developed, taking PAL as independent variable and physical fitness as dependent. A population of 290 medical college freshmen was studied during three consecutive years by directed anamnesis and tests for aerobic and muscular fitness. Collected data were analyzed by linear modeling and Tukey's test, to compare respective averages and Poisson's distribution to effect of dependents variables, according to level of PAL and gender. As main result, highest PAL came along with best fitness scores, indicating adequacy of application of the investigated instrument.

Keywords: level of physical activity, fitness, physical activity, questionnaire.

\section{INTRODUÇÃO}

Atualmente, registra-se na literatura destacado interesse na mensuração da aptidão física $(A F)^{(1)}$, na medida em que significa a capacidade de as pessoas realizarem suas atividades diárias, bem como ocuparem ativamente as horas de lazer e enfrentarem emergências imprevistas sem fadiga excessiva ${ }^{(2)}$. No entanto, a aferição da AF mostra-se tarefa complexa, a qual implica, desde pronto, a definição de quais qualidades físicas a compõem e dos métodos mais adequados(3) 0 primeiro aspecto mencionado tem sido admitido como aparentemente superado, já que força e resistência muscular, capacidade aeróbia, flexibilidade e composição corporal são definidas como as variáveis da AF relacionadas à saúde ${ }^{(4)}$.

Ainda assim, os testes para aferi-la demandam especificidades para execução, fato que vem desafiando outras iniciativas. Nesse sentido, freqüência, intensidade e duração das atividades físicas, habitualmente executadas pelas pessoas, o conhecido nível de atividade física (NAF) têm sido cogitado como estimador da $\mathrm{AF}^{(3)}$.

De fato, é relevante classificar as pessoas em sedentárias ou ativas, pois a inatividade física é admitida como importante fator de risco para doenças crônicas não transmissíveis ${ }^{(3)}$. O sedentarismo pode ser considerado como a ausência ou redução significativa da atividade física, conceito não associado necessariamente à falta de atividade esportiva, mas sim ao hábito da prática de atividade física regular. Consideram-se como sedentárias as pessoas que apresentam pouco gasto energético para realização de suas atividades diárias e ocupação dos momentos de lazer, o equivalente a gasto energético inferior a 500kcal/semana ${ }^{(5)}$. 
De forma geral, os dados disponíveis sobre a prevalência do sedentarismo na América Latina são pouco precisos, pois não existem levantamentos específicos e com metodologias similares e/ou adequadas. Por outro lado, nos Estados Unidos da América (EUA), aproximadamente um em cada quatro adultos refere-se fisicamente inativo (24\%), sobretudo as mulheres respondem ser menos ativas fisicamente (27\%) do que os homens $(21 \%)^{(6)}$. Variações importantes são em relação à etnia, no sentido de que hispânicos apresentaram significativamente maior prevalência de inatividade física (30\%) do que os não hispânicos negros (23\%) ou brancos (20\%). Segundo dados do Departamento de Saúde dos EUA, 40\% dos adultos não realizavam atividade física no tempo livre e somente $15 \%$ deles o faziam moderadamente por 30 minutos ou mais, além de cinco dias na semana $a^{(7)}$.

Especificamente, observa-se certa controvérsia para classificar o NAF das populações. Por exemplo, importante estudo norte-americano ${ }^{(8)}$, visando avaliá-lo nesse âmbito, utilizou-se de questionário para estimar o gasto energético individual em atividades de locomoção (número de quarteirões andados, degraus de escada escalados), esportivas ou recreacionais, e quantidade de horas despendidas em atividades diárias vigorosas, moderadas e leves (ocupacionais ou domésticas), ocorridas durante a semana e ano anteriores. Na mesma linha raciocínio, observa-se, ainda, que o inquérito realizado, nos Estados Unidos em 1988, para subsidiar o Sistema de Vigilância de Fatores de Risco Comportamentais, considerou como vida sedentária menos de três sessões de 20 minutos de AF por semana(9).

Por outro lado, estudo realizado no EUA sobre a manutenção de AF vigorosa em homens e mulheres empregou, ao invés de inquéritos sobre atividades ocupacionais, domésticas e esportivas, a referência da quantidade semanal realizada de exercícios físicos, durante o tempo livre, pelo menos por 20 minutos ininterruptamente, com intensidade suficiente para elevar a freqüência cardíaca e respiratória. Nesse sentido, o NAF foi classificado de acordo com a realização semanal de exercícios vigorosos, nas seguintes categorizações: i) sedentário (nenhuma vez); ii) intermediário (uma ou duas vezes); e iii) ativo (três ou mais vezes) $)^{(10)}$.

Questão relevante é a respeito de qual período de prática de atividade física deve ser adotado, a fim de aceitar o indivíduo como ativo. Em relação a esse aspecto, admite-se a possibilidade de se basear em programas de treinamento como o das Forças Armadas de Cingapura(11) e do Exército Brasileiro(12), os quais usualmente têm a duração de 12 semanas para verificação de adaptações morfo-funcionais da AF, compatível com os três meses preconizados por fisiologistas ${ }^{(13)}$ para ocorrência de alterações fisiológicas e anatômicas no sistema cardiovascular. Ademais, o Colégio Americano de Medicina Esportiva ${ }^{(14)}$ registra que indivíduos que têm participado regularmente nos últimos três meses de exercícios intensos, com no mínimo 15 minutos de duração, três vezes por semana, podem ser tidos como muito ativos.

Finalmente, destaca-se a classificação proposta por Caspersen e Merritt, para definição de: i) inativo - sem tempo definido dedicado a atividade física; ii) ativo irregular - atividade física realizada menos do que três vezes por semana e/ou menos do que 20 minutos por sessão; iii) ativo regular não intenso - três vezes ou mais por semana, 20 minutos ou mais por sessão utilizando menos que $60 \%$ do VO 2 máx; e iv) ativo regular intenso - três vezes ou mais por semana, 20 minutos ou mais por sessão utilizando mais que $60 \%$ do $\mathrm{VO}_{2}$ máx ${ }^{(15)}$.

Entretanto, esses indicadores parecem ser de difícil aplicação na rotina de trabalho dos profissionais da área da Educação Física inte- ressados em identificar e conhecer o NAF das pessoas. Assim sendo, é bastante razoável a adoção de critérios mais flexíveis, levando, principalmente em conta a disponibilidade de tempo, nos dias atuais. Dessa forma, identificações de NAF baseados na tradicional prescrição de três vezes semanais, com sessões de pelo menos 30 minutos, mantendo a freqüência cardíaca na zona aeróbia, pode ser objetivo geral útil na melhoria da AF, mas para muitos indivíduos de nossa sociedade, incluindo jovens estudantes, esse é freqüentemente inatingível(16).

Assim sendo, as recentes recomendações do Colégio Americano de Medicina Esportiva e do Centro de Controle e Prevenção de Doenças indicam a realização diária de ao menos 30 minutos de atividade física moderada, podendo a mesma ser executada de forma cumulativa. Tal prescrição proporciona aos indivíduos: i) maior amplitude para a manutenção de seus programas e ii) alternativa mais atrativa (atividade moderada), especialmente para aqueles que não gostam de praticar exercícios físicos vigorosos ${ }^{(17)}$.

Nesse sentido, o objetivo deste estudo foi verificar a adequação do questionário adaptado de Caspersen \& Merritt (1995) para classificação do NAF em estudantes de Medicina.

\section{MÉTODO}

O delineamento epidemiológico caracterizou-se como transversal seriado(9), uma vez que foram detectadas simultaneamente as variáveis de interesse, em três anos subseqüentes. Como variável independente, identificou-se o nível de atividade física referido e, dependente, a aptidão física.

Tomou-se como população de referência o conjunto dos recémingressos ao curso de Medicina, matriculados na Universidade Estadual de Campinas (Unicamp), em total de 290 pessoas, distribuídas em 146 do sexo masculino $(18,54 \pm 1,21$ anos) e 144 do sexo feminino $(18,46$ $\pm 1,01$ anos), respectivamente.

Após prévio contacto e entendimento pelos responsáveis pela Disciplina de Educação Física Desportiva, componente curricular obrigatório do Curso de Medicina, aplicaram-se a anamnese e os testes de aptidão física durante as próprias aulas, nas dependências da Faculdade de Educação Física da Unicamp. Optou-se por estudar esse grupo específico, justamente devido à oportunidade singular - através da Disciplina de Educação Física Desportiva ministrada pelo Grupo de Saúde Coletiva/Epidemiologia e Atividade Física da Unicamp - de se trabalhar com recém-ingressos ao Curso de Medicina da Unicamp, potenciais formadores de opinião em suas inserções profissionais futuras. Vale dizer que o estudo foi aprovado por Comitê de Ética em Pesquisa, sendo todos os alunos matriculados na disciplina convidados para participar do estudo, destacando-se que, nesse sentido, não houve nenhuma recusa. Contemplado o estabelecido na portaria 196/96 sobre pesquisas em seres humanos no Brasil.

De cada unidade observacional constituinte do grupo mencionado, colheram-se os dados visando, por um lado, através de anamnese dirigida, obter informações a respeito do NAF; por outro, através, dos testes de capacidade aeróbia e muscular, as distribuições das variáveis da AF e de procedimentos biométricos. Para o primeiro procedimento, adaptou-se o já citado critério de Caspersen \& Merritt(15), aplicando-se as seguintes alternativas: i) fisicamente inativo (sem tempo definido dedicado a atividade física); ii) ativo irregular (atividade por menos que três vezes semanais e/ou menos que 20 minutos por sessão) e iii) ativo regular (três vezes ou mais por semana, 20 ou mais minutos por sessão). De fato, o instrumento utilizado procurou atender aos 
três conjuntos de recomendações, especialmente relevantes para a construção de questionários autopreenchidos: i) mostrar-se "amigável" ao respondente, ou seja, envolver, entre outros aspectos, "limpeza", simplicidade, consistência, clareza e parcimônia no uso de perguntas; ii) minimizar o impacto da tendência à leitura parcial de perguntas e opções de resposta, posicionando cláusulas qualificativas ao início das sentenças (e.g. "nos últimos três meses...") e iii) evitar ambigüidade na compreensão das perguntas ${ }^{(18)}$.

As variáveis da AF foram consideradas através dos respectivos procedimentos: i) força/resistência muscular localizada abdominal (RML), medida pelo número de flexões do quadril em 60 segundos ${ }^{(19)}$; ii) força/resistência muscular localizada dos membros superiores (MMSS), caracterizada por flexão/extensão cubital em 30 segundos, com os homens em quatro apoios e a mulheres em seis (20); iii) força dos membros inferiores (MMII) através de teste de salto horizontal(14); e iv) capacidade aeróbia, estimada em teste de pista composto por corrida em 2.400 metros $^{(21)}$. A composição corporal foi quantificada pelo cálculo do índice de massa corporal (IMC) ${ }^{(22)}$, ou seja, o peso corporal dividido pela estatura ao quadrado expresso em $\mathrm{kg} / \mathrm{m}^{2} \mathrm{e}$ categorizada nos seguintes níveis (23): 1) desnutrição (IMC $<18,5$ ); 2) peso adequado (IMC entre 18,6 e 25,0); 3) sobrepeso (IMC entre 25,1 e 30,0); 4) obesidade (IMC 30,1 e 40,0; e 5) obesidade mórbida $($ IMC > 40,1).

A análise estatística foi realizada através do modelo logístico(24) ajustado para IMC, considerando as variáveis NAF, sexo e idade; o teste F (análise de variância), para avaliar consumo de oxigênio segundo NAF e sexo; a prova de Tukey, para comparar as respectivas médias e o modelo de Poisson ${ }^{(25)}$, para verificar o efeito da RML abdominal e força/resistência muscular dos MMSS, segundo nível de atividade física e sexo.

\section{RESULTADOS}

A tabela 1 apresenta a respectiva análise de variância de $\mathrm{VO}_{2}$ máx, e força de MMII segundo NAF e sexo. Observa-se que cada uma dessas variáveis, quando considerada isoladamente, indica associação tanto em relação ao $\mathrm{VO}_{2}$ máx, quanto à força de MMII; no entanto, quando tomadas conjuntamente, tal efeito não se expressa, o que vale dizer que, por exemplo, ativos revelam melhor performance quanto a essas qualidades físicas, independentemente do sexo; da mesma forma que homens o fazem, independentemente do NAF. A tabela 2 informa a comparação das médias de $\mathrm{VO}_{2}$ máx e força dos MMII pelo teste de Tukey, por NAF e sexo: tanto o consumo máximo de oxigênio quanto a força de MMII foram maiores para o grupo ativo e para os rapazes.

Na tabela 3, empregando o modelo de Poisson, analisam-se a RML abdominal e força/resistência dos MMSS, segundo NAF e sexo.

Tabela 1. Análise de variância para $\mathrm{VO}_{2}$ máx e força de MMII, segundo NAF e sexo.

\begin{tabular}{l|c|c|c|c|c|c}
\hline \multirow{2}{*}{ Variáveis } & \multicolumn{3}{c|}{ VO $_{2}$ máx } & \multicolumn{3}{c}{ Força de MMII } \\
\cline { 2 - 7 } & gl & Estatística F & $\boldsymbol{p}$-value & gl & Estatística F & $\boldsymbol{p}$-value \\
\hline NAF & 2 & 26,76 & 0,0001 & 2 & 33,34 & 0,0001 \\
\hline Sexo & 1 & 111,35 & 0,0001 & 1 & 242,40 & 0,0001 \\
\hline Interação NAF e sexo & 2 & 0,91 & 0,4025 & 2 & 0,19 & 0,8288 \\
\hline
\end{tabular}

Tabela 2. Teste de Tukey para médias do $\mathrm{VO}_{2}$ máx. e força de MMII, segundo NAF e sexo.

\begin{tabular}{c|c|c|c}
\hline \multicolumn{2}{c|}{ Variáveis } & VO $_{2}$ máx (ml/kg/min) & Força de MMIl (centímetros) \\
\hline \multirow{3}{*}{ NAF } & 1 & $27,82 \mathrm{~A}$ & $172,12 \mathrm{~A}$ \\
\cline { 2 - 4 } & 2 & $31,57 \mathrm{~B}$ & $192,86 \mathrm{~B}$ \\
\cline { 2 - 4 } & 3 & $36,14 \mathrm{C}$ & $199,34 \mathrm{~B}$ \\
\hline \multirow{3}{*}{ Sexo } & $\mathrm{F}$ & $25,98 \mathrm{~A}$ & $161,28 \mathrm{~A}$ \\
\cline { 2 - 4 } & $\mathrm{M}$ & $35,87 \mathrm{~B}$ & $209,81 \mathrm{~B}$ \\
\hline
\end{tabular}

Letras distintas expressam diferença estatística $(p<0,05)$

Tabela 3. Ajuste do modelo de Poisson da RML abdominal e força/resistência dos MMSS segundo NAF e sexo.

\begin{tabular}{|c|c|c|c|c|c|c|c|}
\hline \multirow{2}{*}{\multicolumn{2}{|c|}{ Variáveis }} & \multicolumn{3}{|c|}{$\begin{array}{c}\mathrm{RML} \text { abdominal } \\
\text { (repetições em } 60 \text { segundos) }\end{array}$} & \multicolumn{3}{|c|}{$\begin{array}{l}\text { Força/resistência dos MMSS } \\
\text { (repetições em } 30 \text { segundos) }\end{array}$} \\
\hline & & estimativa & $p$-value & $\mathrm{TR}^{1}$ & estimativa & $p$-value & $\mathrm{TR}^{1}$ \\
\hline \multirow{3}{*}{ NAF } & 1 & $-0,1472$ & 0,0001 & 0,86 & $-0,2498$ & 0,0001 & 0,78 \\
\hline & 2 & $-0,0293$ & 0,2338 & 0,97 & $-0,1175$ & 0,0012 & 0,89 \\
\hline & 3 & - & - & - & - & - & - \\
\hline \multirow{2}{*}{ Sexo } & $\mathrm{F}$ & $-0,1901$ & 0,0001 & 0,82 & $-0,1000$ & 0,0005 & 0,90 \\
\hline & $M$ & - & - & - & - & - & - \\
\hline
\end{tabular}

1 taxa de repetição

Nesse sentido, destaca-se que, para expressar os resultados dos testes musculares, os escores dos três NAF foram convertidos em taxa de repetição (TR), ou seja, o coeficiente obtido a partir do número de repetições realizadas dividido pelo tempo estimado para cada teste. Especificamente neste estudo, a TR revelou que os indicadores de AF dos ativos irregulares (exceto na RML abdominal) e dos sedentários foram menores que os dos ativos regulares.

A tabela 4 apresenta o ajuste do modelo logístico para IMC considerando NAF, sexo e idade. A respeito da composição corporal, o IMC não se relacionou com o NAF, mas os escores do sexo masculino foram superiores aos do feminino e os dos calouros jovens superiores aos dos mais velhos. De fato, devido à maior quantidade de massa muscular (determinada por reconhecidos fatores biológicos), podese considerar que, em média, os homens são mais pesados do que

Tabela 4. Ajuste do modelo logístico para IMC considerando NAF, sexo e idade.

\begin{tabular}{c|c|c|c|c}
\hline \multirow{2}{*}{ Variáveis } & \multicolumn{3}{|c}{ IMC } \\
\cline { 2 - 5 } \multicolumn{2}{c|}{} & estimativa & $p$-value & OR \\
\hline \multirow{3}{*}{ NAF } & 1 & $-0,1607$ & 0,6107 & 0,85 \\
& 2 & $-0,1636$ & 0,5972 & 0,85 \\
& 3 & - & - & - \\
\hline \multirow{2}{*}{ Sexo } & F & $-1,1786$ & $0,0001^{*}$ & 0,31 \\
\hline \multirow{2}{*}{ Idade } & $M$ & - & - & - \\
\hline
\end{tabular}

${ }^{*}(p<0,05)$ 
as mulheres e, com o passar dos anos, o peso corporal tende a aumentar, porém era de esperar que sedentários possuíssem IMC mais elevados.

\section{DISCUSSÃO}

Os resultados obtidos indicam, desde pronto, que a adoção do instrumento empregado para conhecer o NAF dos universitários foi coerente e compatível, pois as pessoas que referiram maior NAF também apresentaram melhores índices de aptidão física (AF). Por outro lado, já se esperava que a AF dos homens fosse superior à das mulheres ${ }^{(3)}$, pois, devido a fatores fisiológicos como menor tamanho do miocárdio, freqüência cardíaca mais elevada e menor volume de ejeção, é aceitável, ao comparar indivíduos com o mesmo NAF, que o $\mathrm{VO}_{2}$ máx de pessoas do sexo feminino seja inferior ao de rapazes, bem como os níveis mais baixos de andrógenos e, conseqüentemente de massa muscular, podem explicar o desempenho inferior das garotas nos testes de força(26).

Especificamente, quanto à investigação da composição corporal, vale ressaltar o fato de não haver diferença estatística entre o IMC de ativos e sedentários, evidenciando o conceito de estrutura de determinação, também discutido em investigação envolvendo adolescentes obesos(27), para explicar distribuição do peso corporal nos alunos estudados e não somente o efeito de um fator isolado, no caso, a atividade física.

Embora os testes de AF tenham apresentado resultados já amplamente reconhecidos em nossa área, ou seja, que as capacidades físicas de pessoas ativas são significantemente superiores às de inativas ${ }^{(28)}$, revelaram igualmente a aplicabilidade do instrumento para inquérito do NAF referido. Esse aspecto aponta para a possibilidade de utilização do questionário em outros estudos epidemiológicos, considerando-se a dificuldade em estabelecer critérios e mensurar eficientemente o NAF das populações.

De fato, os parâmetros utilizados em muitos estudos não são consensuais, como pode ser observado em diagnósticos populacionais realizados no Chile e Peru(6), os quais revelaram variação importante do NAF, de acordo com a idade cronológica, com os resultados diferenciados na faixa etária dos $19-29$ anos de 78,1\% (Peru) a 89,5\% (Chile), aumentando para 84,2\% e 93,2\%, respectivamente na idade de 30-44 anos; nos maiores de 45 anos, os valores de prevalência da inatividade física atingiram 92,3\% no Peru e 95,6\% no Chile. Especificamente no Peru, o critério de inatividade física utilizado foi a prática de esportes, jogging ou caminhada com freqüência igual a ou menor que duas vezes por mês. No Chile, o critério utilizado foi a realização de atividade física de pelo menos 30 minutos, no mínimo duas vezes por semana. Os dados apresentados na presente investigação sugerem discreto aumento do sedentarismo com o aumento da idade cronológica, especialmente importante redução no percentual de indivíduos muito ativos conforme avança a idade cronológica.

Tendo em conta os critérios de classificação utilizados no estudo mencionado, o percentual de indivíduos do Estado de São Paulo que atingem a recomendação atual de atividade física para promoção da saúde é de 45,7\%. Em relação aos sedentários e irregularmente ativos, a magnitude de pessoas que não cumprem a recomendação atual de atividade física (pelo menos 30 minutos de atividade física por dia) é de aproximadamente 46\%(29).

Por outro lado, destacada iniciativa desenvolvida no Brasil(30) validou respectivo questionário internacional de atividade física em amostra de adultos jovens brasileiros, através da estimativa de gasto energético semanal. Os principais resultados encontrados apontam que as atividades físicas realizadas durante o trabalho $(82,4 \mathrm{kcal} / \mathrm{kg} / \mathrm{sem})$ representam os maiores gastos energéticos semanais, seguidos pela prática de recreação, esporte e lazer (78,2kcal/kg/sem), sendo as atividades domésticas (26,8kcal/kg/sem) e de locomoção (24,2kcal/kg/sem) aquelas que menos contribuem com o dispêndio total de quilocalorias semanal. Entretanto, a estimativa do gasto energético individual muitas vezes depende de cálculos específicos para a sua realização, sendo baseada em realidades internacionais.

Considerando as limitações de identificar o NAF através de mensurações ou estimativas de gasto energético, o NAF referido em questionário parece ser ferramenta eficiente para classificar a atividade física habitual das pessoas. Nesse sentido, os respectivos resultados encontrados, no presente estudo, revelam a adequação da aplicação do questionário adaptado de Caspersen \& Merritt, como instrumento viável para obtenção do NAF, através da aplicação de inquérito simples e de rápida aplicação.

Contudo, devem-se atender os apontamentos de autores ${ }^{(31)}$ que mencionam a atenção especial a ser centrada nos aspectos de padronização e validação dos questionários, ao ressaltarem que uma única folha pode conter princípios epidemiológicos e ser administrada em grandes populações. Sobretudo, tem-se buscado mensurar a aptidão aeróbia através de testes "sem-exercício", com a finalidade de agilizar a quantificação da aptidão cardiorrespiratória em estudos populacionais ${ }^{(32)}$.

No entanto, como já mencionado, existem ainda dificuldades em avaliar as atividades físicas menos vigorosas ${ }^{(33)}$ - trabalhos domésticos, lazer, caminhada para o escritório, entre outras - as quais também podem exercer efeito protetor para redução de doenças cardiovasculares ${ }^{(34)}$. Fato esse que também não foi observado no presente estudo.

Em contrapartida, parece ser mais preciso identificar os exercícios vigorosos, pois são usualmente realizados com freqüência e duração determinadas durante a semana, facilitando o registro, como o observado na presente investigação.

\section{CONCLUSÃO}

Finalmente, a partir dos dados coletados, pode-se concluir que o questionário adaptado de Caspersen e Merritt utilizado para obter o NAF de estudantes de Medicina mostrou-se adequado. Uma vez que os indivíduos que referiram maior NAF, de fato apresentaram aptidão física superior quando comparados com os demais. Assim sendo, o respectivo questionário adaptado, pode ser empregado em outras iniciativas envolvendo populações com características semelhantes.

Todos os autores declararam não haver qualquer potencial conflito de interesses referente a este artigo. 
1. Conte $M$, Gonçalves A. Avaliação somática e funcional em mulheres ativas: relações entre as variáveis da aptidão física e cardiovasculares. Salus 1997;16:63-76.

2. Guedes DP, Guedes JERP. Exercício físico na promoção da saúde. Londrina: Midiograf, 1995

3. Conte M, Gonçalves A. Qualidade de Vida e aptidão física. In: Gonçalves A, Vilarta R, editores. Qualidade de vida e atividade física. Barueri: Manole, 2004;257-87.

4. American College of Sports and Medicine. The Recommend Quantity and Quality of Exercise for Developing and Maintaining Cardiorepiratory and Muscular Fitness, and Flexibility in Healthy Adults. Med Sci Sports Exerc 1998;30:975-1.

5. Nahas MV. Atividade física, saúde e qualidade de vida: conceitos e sugestões para um estilo de vida ativo. Londrina: Midiograf, 2003.

6. Matsudo SM, Matsudo VR, Araújo T, Andrade D, Andrade E, Oliveira L, et al. Nível de atividade física da população do estado de São Paulo: análise de acordo com o gênero, idade, nível sócio-econômico, distribuição geográfica e de conhecimento. Rev Bras Ciência Mov 2002;10:41-50.

7. United States Department of Health \& Center Disease Control. Increasing Physical Activity: A Report on Recommendations of the Task Force on Community Preventive Services. MMWR Recomm Rep 2001;50:1-14.

8. Cauley JA, Donfield SM, LaPorte RE, Warhafting NE. Physical activity by socioeconomic status in two populations based cohorts. Med Sci Sports Exerc 1991;23:343-52.

9. Pereira MG. Epidemiologia: teoria e prática. Rio de Janeiro: Guanabara Koogan, 1995.

10. Sallis JF, Hovell MF, Hofstetter CR. Predictors of adoption and maintenance of vigorous physical activity in men and women. Prev Med 1992;21:237-51.

11. Lim CL, Lee LKH. The effects of 20 weeks basic military training program on body composition, $\mathrm{VO}_{2}$ max and aerobic fitness of obese recruits. J Sports Med 1994;34:271-7.

12. Ministério do Exército. Manual de Campanha:Treinamento Físico Militar. Brasília: Ministério do Exercito, 1990.

13. Leite PF. Fisiologia do exercício: ergometria e condicionamento físico. São Paulo: Atheneu, 1986.

14. Colégio Americano de Medicina Esportiva. Guia para Teste de Esforço e Prescrição de Exercício. Rio de Janeiro: Medsi, 1987.

15. Caspersen CJ, Merritt RK. Physical activity trends among 26 states, 1986-1990. Med Sci Sports Exerc 1995;27:713-20

16. Kig AC, Martin JE. Aderência ao exercício. In: American College of Sports Medicine. Prova de Esforço e Prescrição de Exercício. Rio de Janeiro: Revinter, 1994;332-41.
17. Blair SN. Exercise prescription for health. Quest 1995;47:338-53

18. Faerstein E. Pré-testes de um questionário multidimensional autopreenchido por população adulta: a experiência do pró-saúde UERJ. In: Congresso Brasileiro de Saúde Coletiva, 6, Salvador-BA, setembro de 2000.

19. Mathews DK. Medida e avaliação em educação física. Rio de Janeiro: Guanabara, 1986.

20. Kiss MAPDM. Avaliação em educação física. São Paulo: Manole, 1987.

21. Cooper K. O programa aeróbico para o bem-estar total. Rio de Janeiro: Nórdica, 1982.

22. McArdle WD, Katch FI, Katch VL. Fisiologia do exercício: energia, nutrição e desempenho humano. Rio de Janeiro: Guanabara Koogan, 2004.

23. World Health Organization Expert Committee. Physical Status: The Use and Interpretation of Anthropometry. Geneva: WHO, 1995.

24. McCullagh P, Nelder JA. Generalized linear models. London: Chapman \& Hall, 1989

25. McCullagh P. Regression models for ordinal data (with discussion). JSTOR 1980;42:109-42.

26. Mellion MB. Segredos em medicina desportiva: respostas necessárias ao dia-a-dia em centros de treinamento, na clínica, em exames orais e escritos. Porto Alegre: Artes Médicas, 1997.

27. Conte $M$, Gonçalves A, Aragon FF, Padovani CR. Influência da massa corporal sobre a aptidão física em adolescentes: estudo a partir de escolares do ensino fundamental e médio de Sorocaba/SP. Rev Bras Med Esporte 2000;6:44-9.

28. Karvonen MJ. Physical activity for a healthy life. Res Q Exerc Sport 1996;67:213-5

29. Matsudo VKR, Matsudo SMM, Andrade D, Araújo T, Andrade E, Oliveira LC, et al. Promotion of physical activity in a developing country: The Agita São Paulo Experience. Public Health Nutr 2002;5:253-61

30. Barros MVG, Nahas MV. Reprodutividade (teste-reteste) do questionário internacional da atividade física (QIAF-Versão 6): um estudo piloto em adultos. Rev Bras Ciência Mov 2000;1:23-6.

31. Paffenbarger RS, Blair SN, Lee IM, Hyde RT. Measurement of physical activity to assess heatlh effects in free-living populations. Med Sci Sports Exerc 1993;25:60-70.

32. Whaley MH, Kaminsk LA, Dwyer GB, Getchell LH. Failure of predicted $\mathrm{VO}_{2}$ peak to discriminate physica fitness in epidemiological studies. Med Sci Sports Exerc 1995;27:85-91.

33. Lee IM, Paffenbarger JR R. How much physical activity is optimal for health? Methodological considerations. Res Q Exerc Sport 1996;67:206-8.

34. McMurray RG, Ainsworth BE, Harrel JS, Griggs TR, Williams OD. Is physical activity or aerobic power more influential on reducing cardiovascular disease risk factors? Med Sci Sports Exerc 1998;30:1521-9. 